
cultures

Les cahiers de l'Acedle

1 | 2004

Actes de la journée Métice

\title{
L'apport original du forum de discussion de WebCT dans un cours d'anglais appliqué à l'archéologie
}

Joseph Rézeau

\section{OpenEdition}

12 Journals

Édition électronique

URL : http://journals.openedition.org/rdlc/5399

DOI : $10.4000 /$ rdlc.5399

ISSN : 1958-5772

Éditeur

ACEDLE

\section{Référence électronique}

Joseph Rézeau, «L'apport original du forum de discussion de WebCT dans un cours d'anglais appliqué à l'archéologie », Recherches en didactique des langues et des cultures [En ligne], 1 | 2004, mis en ligne le 15 novembre 2004, consulté le 11 septembre 2019. URL : http://journals.openedition.org/ rdlc/5399; DOl : 10.4000/rdlc.5399

Ce document a été généré automatiquement le 11 septembre 2019.

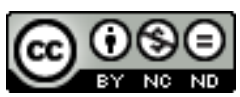

Recherches en didactique des langues et des cultures is licensed under a Creative Commons AttributionNonCommercial-NoDerivatives 4.0 International License 


\title{
L'apport original du forum de discussion de WebCT dans un cours d'anglais appliqué à l'archéologie
}

\author{
Joseph Rézeau
}

\section{Introduction}

1 Au moment où nombre d'institutions de l'enseignement supérieur en France mettent en place des cours en ligne, on peut s'interroger sur la pertinence des nouveaux outils d'apprentissage dits collaboratifs tels que le clavardage (chat) ou les forums de discussion et leur apport spécifique par rapport au cours en présentiel.

2 Plus précisément, dans le cadre de l'apprentissage d'une langue étrangère par des étudiants spécialistes d'autres disciplines, on peut espérer que l'outil "forum de discussions" - utilisé en complément d'autres outils plus traditionnels disponibles sur la plate-forme - offrira aux apprenants la possibilité non seulement d'être exposés à des stimuli en langue cible mais surtout de produire eux-mêmes de la langue cible.

En effet, les théories actuelles de l'apprentissage des langues mettent l'accent sur l'utilisation de la langue cible comme moteur d'une réflexion linguistique propice à l'apprentissage.

4 Nous commençons par présenter le cadre général dans lequel se situe l'expérience menée avec nos étudiants d'archéologie à l'université de Rennes 2.

5 Dans notre deuxième point, nous suivons la typologie proposée par Lamy \& Goodfellow (1998) pour classer et donner des exemples de ce que ces auteurs appellent des "monologues", des "conversations socio-affectives" et des "dialogues réflexifs". Nous cherchons ensuite à savoir si le dispositif que nous avons mis en place suscite le troisième type d'échanges, celui des dialogues réflexifs, considéré par ces auteurs comme le plus favorable à l'acquisition de la langue étrangère. 


\section{Le dispositif}

6 Le cours d'anglais appliqué à l'archéologie est destiné à des étudiants de niveau licence (troisième année d'université) qui ont suivi les deux années précédentes un enseignement d'anglais spécialisé "histoire de l'art et archéologie" à l'université de Rennes 2. Ce cours est donné en présentiel, à raison de 3 heures hebdomadaires consécutives regroupées sur le deuxième semestre de l'année universitaire. L'objectif prioritaire du cours est l'appropriation d'outils favorisant l'autonomie des étudiants dans la lecture et la compréhension écrite de documents d'archéologie en langue anglaise. En parallèle, le cours d'anglais est accessible sur la plate-forme WebCT, où les étudiants retrouvent des textes à lire et des exercices linguistiques, similaires à ou complémentaires de ceux étudiés en classe. Sur WebCT, les étudiants ont également accès à un forum de discussion. Le dispositif est donc du type "apprentissage mixte" (ou blended learning).

7 Ce cours étant dispensé au deuxième semestre de l'année universitaire, nous ne disposons pas encore - au moment de rédiger cet article - de la totalité des données de l'année en cours, et notre étude portera donc sur les données de l'année 2002-2003. En fin de semestre (fin mai 2003), une enquête a été réalisée, à laquelle 23 des 30 étudiants concernés ont répondu. Nous allons extraire des résultats de cette enquête quelques éléments significatifs et les commenter.

\subsection{Cohérence et objectifs}

8 La toute première question de notre enquête portait sur la cohérence entre les deux éléments mis en œuvre dans le dispositif d'apprentissage mixte, à savoir le cours en présentiel et le cours à distance (Figure 1a). On constate que les étudiants sont très majoritairement d'avis que le dispositif est cohérent ; on ne relève aucune réponse négative. Quant aux $35 \%$ d'étudiants qui ne se sont pas prononcés (NSP), on peut interpréter leur réserve soit comme une incompréhension du concept de cohérence (dans ce contexte) soit comme un refus de répondre à une question dont ils estiment qu'elle s'adresse prioritairement à l'enseignant et pas à l'apprenant.

9 La question suivante demandait d'apprécier la pertinence du cours à distance par rapport à un objectif d'autonomisation de l'apprentissage. En réalité, cet objectif n'a jamais été déclaré comme tel dans la définition du cours ; mais nous considérons qu'il était sousjacent au dispositif mis en place (Figure $1 \mathrm{~b}$ ). La faible proportion de réponses négatives nous incite à penser que la majorité des sujets ont intégré la dimension autonomisante du dispositif. 
Figure 1 - Cohérence du dispositif et autonomie.

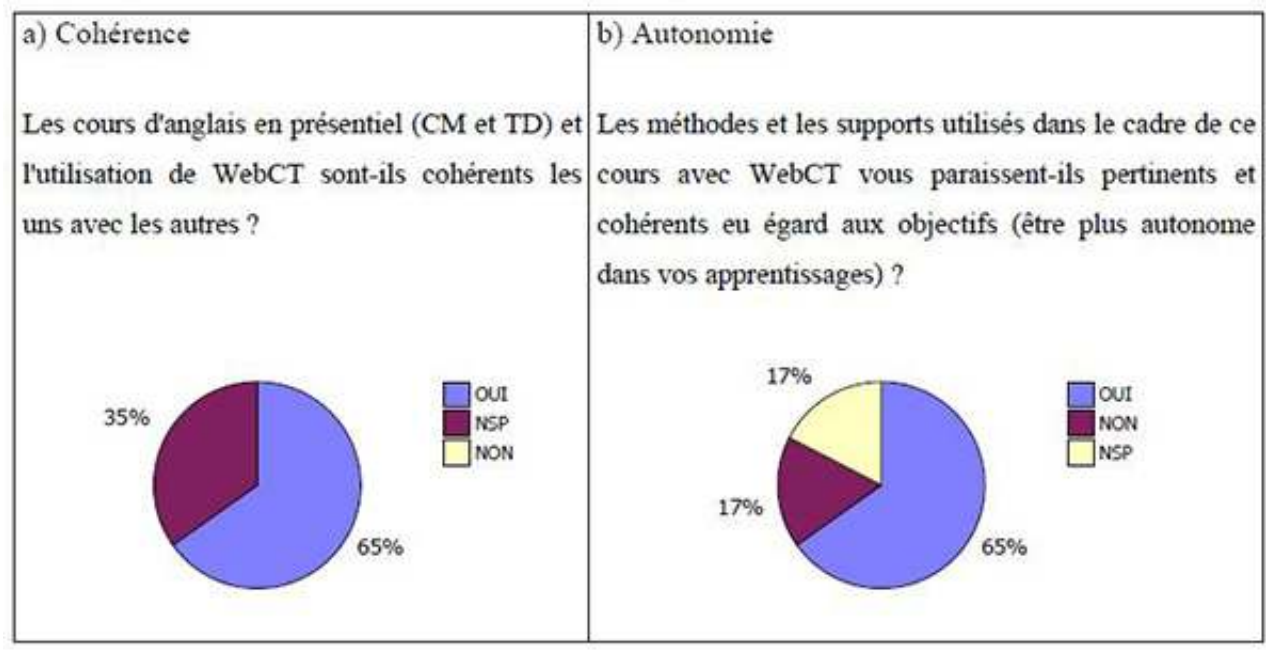

\subsection{Niveau de compétence technique}

Dans la mesure où l'utilisation optimale d'une plate-forme d'enseignement à distance requiert un niveau minimum de compétences techniques, il nous a semblé intéressant de chercher à connaître l'opinion de nos étudiants sur leurs propres compétences, à l'issue de l'expérience (Figure 2). Les réponses sont sans surprise : les sujets concernés possèdent un niveau convenable à très bon dans le domaine de la navigation sur Internet. Les données des réponses $\mathrm{d}$ ) et surtout e) sont un reflet du manque de convivialité des outils "messagerie" et "discussions" de la plate- forme WebcT.

Figure 2 - Niveaux de compétences.

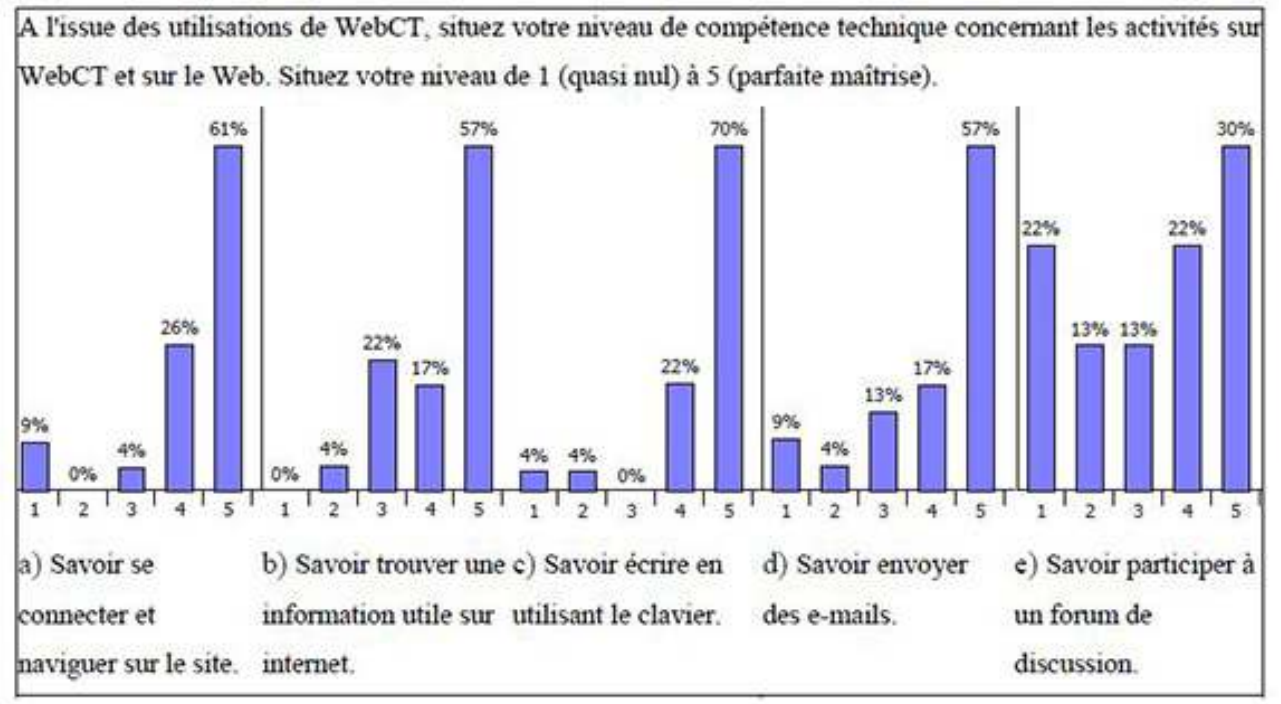




\subsection{Formation et usages}

11 Les réponses aux questions a) et b) portant sur les lieux de formation (Figure 3) sont tout à fait caractéristiques de l'usage actuel des TICE (Technologies de l'Information et de la Communication pour l'Enseignement) dans notre pays. Tout comme leurs enseignants, nos étudiants ne bénéficient pas (ou peu) d'une formation à l'utilisation des outils informatiques : la formation se fait dans la vie quotidienne. Par ailleurs, le cours en ligne est encore une rareté dans le type d'institution où nous travaillons (une université française de lettres et sciences humaines). Comme toujours, il y a loin des discours politiques à la réalité.

Figure 3 - Lieux de formation.

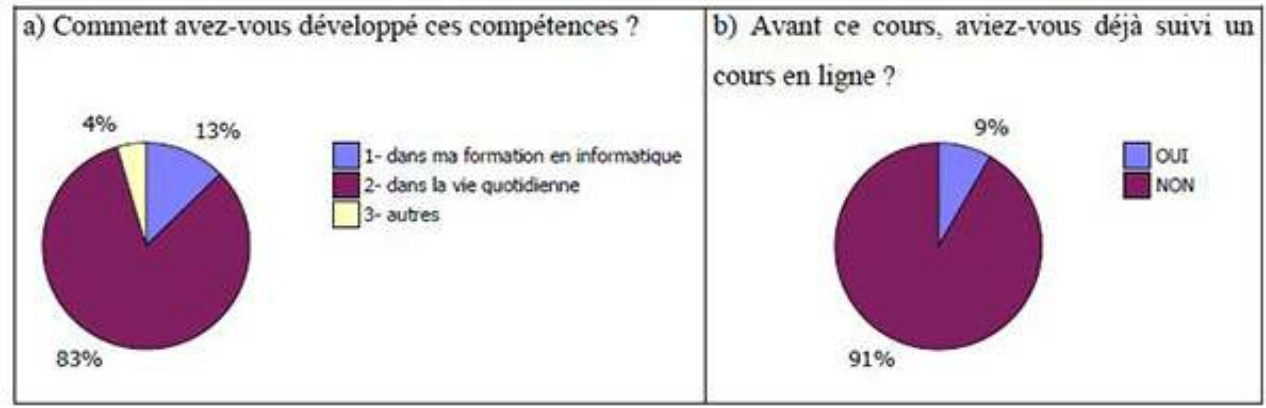

L'accès aux cours à distance est à peu près également réparti entre l'institution et le domicile des étudiants (Figure 4a). Tandis que pour suivre les cours magistraux et travaux pratiques les étudiants doivent se présenter sur le campus de l'université, la plate-forme d'enseignement à distance leur laisse le choix du lieu d'accès. Les principales raisons qui motivent un accès autre que sur le campus sont - en ce qui concerne notre propre institution - le nombre insuffisant des salles informatiques, les horaires restreints aux "jours et heures ouvrables" et la vétusté ou le manque de périphériques (imprimantes, multimédia) des ordinateurs mis à la disposition des étudiants. Par ailleurs, la montée en puissance de l'équipement personnel des étudiants ou de leur famille, de l'accès rapide à Internet (ADSL) sont des facteurs qui facilitent et incitent les accès hors de l'institution.

En ce qui concerne le temps passé sur la plate-forme par semaine (Figure 4b), il s'agit d'une indication à prendre avec précaution. Le système de suivi (ou tracking) incorporé à WebCT permet de savoir exactement quand un étudiant "entre" sur une certaine page d'un cours, mais pas quand il quitte le cours ; en outre, il n'y a pas d'outil permettant facilement de totaliser le temps passé par un étudiant sur un cours donné. 


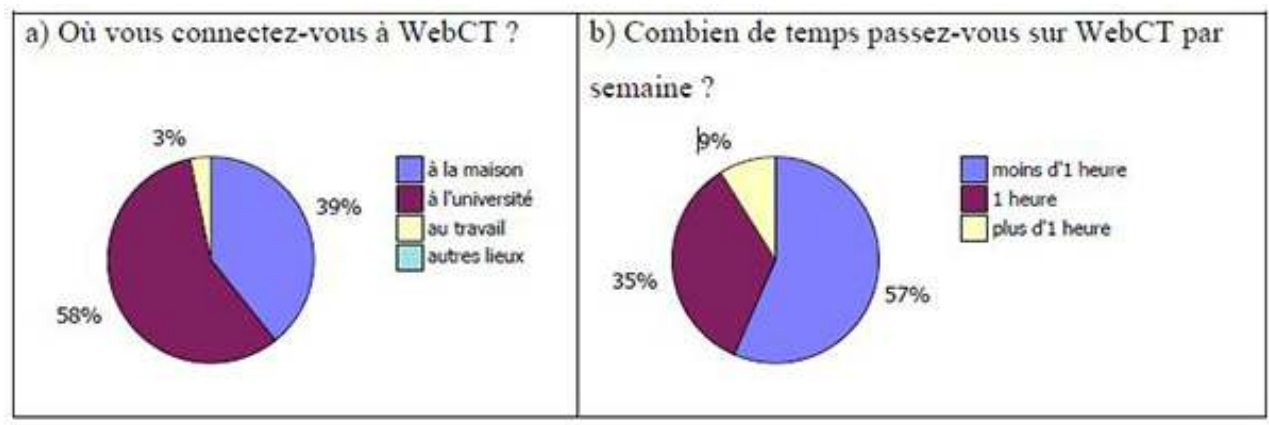

14 Parmi les divers outils à la disposition des étudiants pour ce cours d'anglais sur WebCT, on remarquera que les plus utilisés sont précisément ceux qui ne font pas double emploi avec les activités en présentiel et en premier lieu le forum de discussions, objet principal du présent article (Figure 5).

Figure 5 - Réponses données à la question : "À quelles activités participez-vous sur le cours WebCT ?"

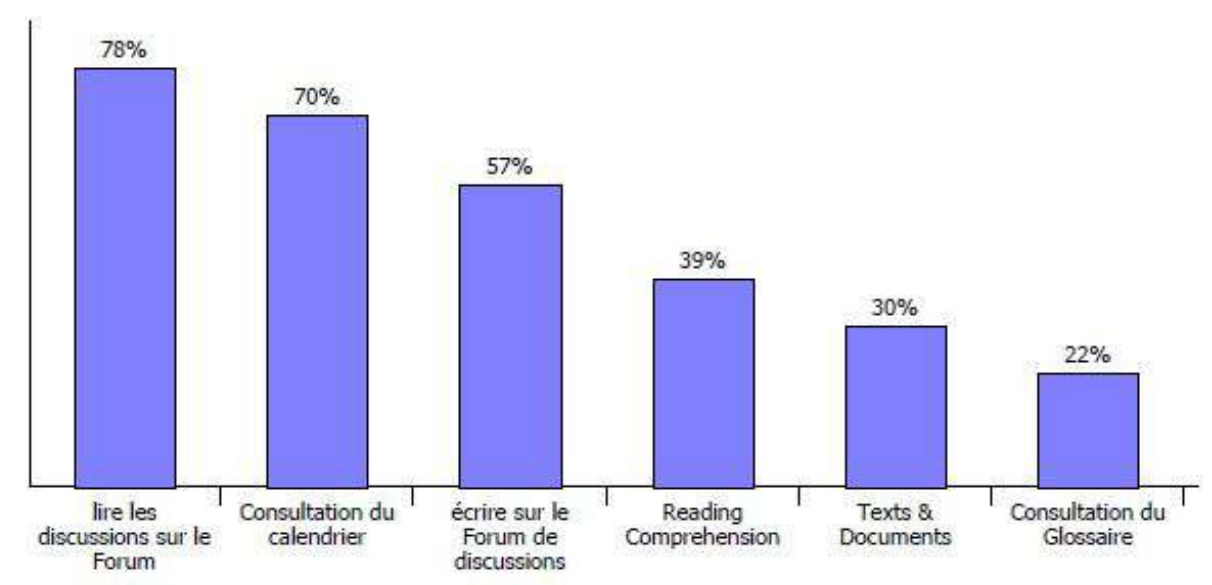

\section{Le forum de discussion : monologues, conversations et dialogues}

Dans leur article de 1998, Lamy \& Goodfellow empruntent à van Lier son modèle socioaffectif de l'interactivité. Selon ce dernier auteur, les modalités de l'échange enseignantapprenant vont de l'extrémité la plus "contrôlante", par exemple le "monologue" (du professeur donnant son cours magistral), pendant lequel l'élève se tait, au "dialogue" (entre professeur et élève dans la salle de classe) dans lequel la parole passe de l'un à l'autre mais toujours sous le contrôle du professeur, pour aller jusqu'à la "conversation", dans laquelle la communauté des apprenants explore librement la connaissance, sans que le plan préalable du maître influe sur le cours des événements (van Lier, 1996 : 180-181).

Lamy \& Goodfellow ont transposé au domaine des discussions sur une plate-forme d'apprentissage en ligne ces trois types d'échanges pour caractériser les messages échangés par les participants. Nous utiliserons leur modèle et leur terminologie pour l'appliquer à l'analyse d'échantillons d'échanges prélevés dans le corpus rassemblé lors 
de notre expérience. Nous rappelons ci-dessous la terminologie de Lamy \& Goodfellow ( op. cit.: 87).

- le "monologue" est un message solitaire - qui peut avoir été inspiré par des messages postés par d'autres participants, mais il ne fait pas référence à ceux-ci, et ne requiert ni ne suggère de réponse. Il n'est donc pas considéré comme "interactif" ;

- le "dialogue réflexif" s'applique aux interactions impliquant une réflexion sur la langue ;

- enfin le terme "conversation" désigne des échanges de type purement socio-affectif.

\subsection{Le monologue}

\subsubsection{Consignes}

17 Chaque message ou extrait de message posté sur le forum de discussion du cours d'anglais pour archéologie est cité ci-après selon le format suivant : numéro d'ordre du message ; éventuellement numéro d'ordre du message original auquel il répond ; date et heure ; nom de l'expéditeur ; titre éventuel ; corps du message ou extrait. Pour respecter la vie privée des participants, le prénom a été conservé mais le patronyme réduit à sa lettre initiale.

Msg. $n^{\circ} 4$--- 05/02/2003 19:10.

Joseph Rézeau Welcome to the Archaeology Discussion List.

Hello everybody! Welcome to this Discussion List where you will be able to post any message relevant to your English Course for Archaeology students.

----- RULES -----

1- All messages should be posted in English. 2- Messages must deal with topics relevant to the English language, archaeology, as well as more general topics of interest (...).

Ce message est une consigne, il ne s'adresse à personne en particulier et ne réclame aucune réponse. Bien qu'il ait son utilité (pour le fonctionnement du dispositif) son manque d'interactivité inhérente entraîne un rôle minimal pour l'acquisition de la langue.

\subsubsection{Messages "hors normes"}

Msg. $n^{\circ} 27$ Branch from $n^{\circ} 0024$ 12/02/2003 18:03 SIMON P.

Soy de acuerdo con vosotros pienso que falta practica en nuestras estudias . He olvidado un poco mi espanol y Jose va a reir al leer eso.Se puede que he olvidado tambien la lengua que debiamos emplear para escribir los mensajes, voy a terminar ahora.

Msg. $n^{\circ} 87$ Branch from $n^{\circ} 0027$ 11/03/2003 21:53 SIMON P.

Penn disonj ! N'eo ket diaes memes tra! Dav eo skrivan e saozneg, saozneg ha net ra all ! Petramant , 'vo an ael du ha plac'h al levriou (e galleg )o c'hoarzin diouzhit! It's the last time I tell you!

Un étudiant "original" a posté des messages en langues étrangères autres que l'anglais. Personne d'autre que lui-même n'y a répondu. En réalité, ce monologue a quand même eu 
des suites. J'avais envoyé un message privé à l'auteur pour lui demander des explications ; voici ce qu'il a posté sur la liste.

Msg. n 93 Branch from nº 0087 17/03/2003 11:51 SIMON P.

Mr Rézeau asked me to translate my breton message into english but I'm afraid it would loose its real signification and interest translated into an other language . I just told myself that english and only english was the language I could use,to reply to my spanish message. Eso es toud folks !

\subsubsection{Pseudo-dialogue à la Monty Python}

Message $n^{\circ} 28$ 12/02/2003 18:28 JEROME C. monty python

If you like the monty python, this message is for you ! Thursday 13th, There's "the quest for the holy grail" on arte. Next week it will be "brian's life" ! (...)

Message $n^{\circ} 51$ JEROME C.

Oh I'm scared ! bouh ! oh ! Let me laught ! Ah ah ah ! Well... it's time to go home.

Hum... sorry... for all.

Cet étudiant a ainsi posté une douzaine de messages, se répondant à lui-même. Je dois avouer que je n'ai pas tout de suite compris l'humour de la démarche de Jérôme, typique des Monty Python. J'ai donc effacé ses messages du forum et lui ai envoyé un message en privé pour l'en avertir. Il m'a alors expliqué la référence au pseudo-dialogue/ monologue...

\subsection{La conversation}

\subsubsection{Is the English lesson really English?}

Msg. $n^{\circ} 5$ 07/02/2003 09:29 MYRIAM M. first english lesson.

Hello! I don't know if the others students have the same sensation as me, but during the first lesson I wasn't sure that I was in an ENGLISH lesson. Maybe it's because the instructions about Netscape Composer weren't in english, but I hope the next lesson will be more about english. I really believe, Mr Rézeau, you can speak more in english during the lessons (but maybe the others disagree with me...), even if it makes things more difficult to us.

Nous avons dans ce message plusieurs caractéristiques de la conversation (interactive) :

- les réactions des autres participants sont sollicitées (...the others students) ;

- les allocutaires sont clairement signalés (Mr Rézeau);

- les précautions oratoires sont de mise (Maybe it's because... ; maybe the others...; even if...).

D'ailleurs ce premier message a bien été productif puisqu'il a suscité un total de 6 réactions (ci- après).

Msg. $\mathrm{n}^{\circ} 6$ Branch from $\mathrm{n}^{\circ} 0005$ 07/02/2003 10:12 Joseph Rézeau re: first english lesson.

Thank you Myriam for your judicious remarks. I quite agree with you that there was not a lot of English spoken in that first English lesson... Don't worry, in the next lessons we will be using more English, and of course in the second part of the lesson, all the instructions about using the software will be in English! 
La personne attaquée répond : elle admet volontiers les remarques faites par son adversaire (I quite agree... judicious remarks...) ; elle rassure à propos de la suite des événements...

Msg. $n^{\circ} 7$ Branch from n ${ }^{\circ} 0005$ 07/02/2003 11:12 ELISE B. re: first english lesson.

so am I, it was a little like for children....But we must think that we know mister Rezeau, but few people come from other university, so, prhaps it is better beginning like this. I think it is not very dramathic. :-). We 'll see to the next lesson!! Bye. Dans cette réaction, Elise exprime son accord avec l'auteure du message original (so am I), elle prend la défense de la personne "attaquée" (we know mister Rézeau) ; elle se met à sa place pour expliquer sa stratégie par rapport aux "nouveaux" étudiants ; enfin elle minimise l'importance de l'événement et conseille de patienter jusqu'au prochain cours!

Msg. $n^{\circ} 8$ Branch from n ${ }^{\circ} 0007$ 10/02/2003 08:34 MYRIAM M. re: first english lesson.

I agree with you both and I will wait the next lesson, perhaps my critics will not be true. Mr Rezeau, I have found a book about flint knapping, and in this book thre is a small list of vocabulary about tools (english-french and french-english), it's very interesting. I haven't got it with me but if you want, I will give you references thurday.

L'auteur du message original, Myriam, répond au professeur et à sa camarade Elise. Elle accepte de nuancer ses critiques. Pour se "racheter" auprès de la personne accusée, elle lui fait un petit cadeau : elle propose de lui fournir les références d'un ouvrage sur un thème abordé en cours (flint-knapping)...

Msg. n 9 --- 10/02/2003 10:57 LAURENCE LE C. a little discussion!!!!

hello!!!!!!!!!!!! About next lesson:I think it was interresting to learn how creat a text with links but as the others it's seems to be more a lesson of internet. But, to open the discussion, we just had a lesson about Middle Age in Britany and does someone know something about Sutton Hoo? It's seems to be a famous grave of the Dark Ages.

Le message $\mathrm{n}^{\circ} 9$ met fin à la conversation en cours. Laurence apporte sa contribution au débat, en pesant le pour et le contre, puis propose de passer à autre chose (But, to open the discussion (...).

\subsubsection{We must fight for archeology!}

Msg. n 56 --- 14/02/2003 13:16 DELPHINE B. about archeology

Hello students! I would like to tell my point of wiew about archeology's situation in this moment. I fell very desapointed when I see the governement whant to close lot of sites, because I think all informations that we could study will go out (...) Without want to talk about politic, I just want to tell I'm sad and angry (...) Is there any hope?

Msg. $n^{\circ} 59$ Branch from $n^{\circ} 0056$ 17/02/2003 11:20 LAURENCE LE C. about archeology

Dear Delphine!!!!!!!!! Don't be so desapointed!!oK, today, in our country, it's not easy when you want to pratice your favorite activity:archeology and it will probably be worse later!!!BUT you have to keep your smile because we must fight for our futur! (...) laurence.

Msg. $\mathrm{n}^{\circ} 60$ Branch from nº 0059 17/02/2003 12:23 MYRIAM M. about archeology. 
Laurence, I totally agree with you when you say we must fight for our future!!! (...)

We must fight for archeology!!!

Msg. $n^{\circ} 63$ Branch from nº 0061 17/02/2003 12:36 MYRIAM M. about archeology

Yes, it's a politic problem, and I'm very angry by a lot of things in politic today...

But this forum is not the place to speak about that (...).

Les extraits ci-dessus ne sont qu'une petite partie d'une longue discussion asynchrone qui a eu lieu peu après le début des séances de cours d'anglais, en février 2003. Cette discussion a impliqué 8 étudiants, qui ont posté un total de 14 messages, et s'est déroulée sur 5 jours. La défense de l'archéologie est bien entendu un sujet d'une importance capitale pour les étudiants en question, et cette importance sociale du sujet explique la véhémence des propos. A propos d'exemples de conversations de même type, Lamy \& Goodfellow écrivent :

selon nous, bien que ces échanges soient indubitablement interactifs au sens où on l'entend dans le modèle socio-affectif de l'interactivité (égalité des participants, partage des tours de parole, choix des thèmes établi collectivement), la nature asynchrone du support milite contre l'acquisition telle que le modèle cognitif l'envisage. En effet, cette conversation est étalée sur six jours, et vu la relative superficialité du contenu, on imagine mal comment pourraient intervenir la négociation du sens ou l'attention portée à la forme (Lamy \& Goodfellow, 1998 : 88).

Nous partageons globalement ce point de vue, mais au vu des exemples cités plus haut, il ne nous paraît pas flagrant que la nature asynchrone de la discussion empêche négociation du sens ou attention portée à la forme. Nous avons un bon exemple de reprise d'une forme incorrecte (we must fight for our futur) du message 59 et sa correction dans le message 60 .

\subsection{Le dialogue réflexif}

Notons tout d'abord que, dans l'expérience rapportée par Lamy \& Goodfellow, deux forums en langue cible étaient mis à la disposition des étudiants : l'un dédié aux échanges de type linguistique, l'autre, appelé "le café", libre ... Les exemples de ce que ces auteurs appellent des dialogues réflexifs se trouvent dans le premier forum.

Dans le cadre de mon cours d'anglais appliqué à l'archéologie j'ai ouvert un forum unique, les exemples de dialogues réflexifs se trouvent mélangés aux conversations et (est-ce une conséquence ?) leur nombre est relativement restreint.

31 Pour Lamy \& Goodfellow, les dialogues réflexifs sont ceux qui se réfèrent explicitement à la langue et à son apprentissage. Ces auteurs soutiennent la thèse que ce sont essentiellement les dialogues de ce type qui favorisent l'acquisition de la langue.

\subsubsection{L'autocorrection}

Msg. $\mathrm{n}^{\circ} 30$ Branch from $n^{\circ} 0005$ 12/02/2003 18:42 HELENE M. re: first english lesson

i'm very disappointed because the first english was not in english... I shed a tear on this sad event

Msg. $n^{\circ} 53$ Branch from $n^{\circ} 0030$ 12/02/2003 20:32 HELENE M. re: first english lesson the first english LESSON! scuse me, I forget my latin. 
Ce n'est pas ici le lieu de s'étendre sur les vertus de l'autocorrection. Cet exemple montre simplement que ce phénomène existe bel et bien, et qu'il apparait indépendamment du dispositif d'enseignement-apprentissage mis en œuvre ... Notons que l'étudiante s'est aperçue de son erreur en relisant son message à peine deux heures plus tard, le même jour. Notons également que son second message comporte : la correction, des excuses et même une pointe d'humour.

\subsubsection{Hétéro-correction entre pairs et reprises "congratulatoires"}

\section{Reprise avec correction}

Msg. $\mathrm{n}^{\circ} 126$ 03/04/2003 19:01 NATACHA J. About quarrels between research workers.

I was present at a medieval symposium the last week-end and like there were few research workers, historians or archeologists, maybe about ten, certains was at odds between us. It is comprehensible that discussions born during this type of symposium. It's necessary but some quarrels are like "disputes of supermarket" as i say sometimes and they don't to advance research (...) What do you think of that?

Msg. $\mathrm{n}^{\circ} 129$ Branch from $\mathrm{n}^{\circ} 0126$ 07/04/2003 08:35 MYRIAM M. re: About quarrels between research workers.

I totally agree with you, there's a big difference between a scientific discussion and a "supermarket quarrel" (I didn't know this expression!!!), and it's very bad because there's always the students who have embarrassed with that...

Dans cet échange on note que Natacha invente de toutes pièces une expression pour traduire "querelle de clocher" ou de comptoir ; elle s'en attribue d'ailleurs la paternité (as i say sometimes). Dans sa réponse, Myriam adopte l'expression forgée par sa camarade, en la modifiant toutefois, probalement pour lui donner une allure qu'elle juge plus anglaise ( supermarket quarrel).

\section{Reprise à l'identique}

Msg. $\mathrm{n}^{\circ} 64$ Branch from nº 0056 17/02/2003 19:40 HELENE M. about archeology.

yes! we must go on the steet to fight against this business power. for singing tomorrow, archeologist of all the nation wake up!! under the macadam the sites! (...)

Msg. $n^{\circ} 68$ Branch from nº 0064 19/02/2003 08:27 MYRIAM M. about archeology (...) By the way, I really like your little sentence : "under the macadam the sites"!!!

Dans cet échange, Myriam complimente sa camarade Hélène sur sa trouvaille, calquée sur le fameux "sous les pavés la plage", mais n'en propose pas de correction, difficile d'ailleurs à trouver...

\section{Erreur linguistique ou factuelle?}

Msg. n 124 --- 03/04/2003 08:26 MYRIAM M. SAO...

(...) on Tuesday the 8th there is the last "West Archeological Seminary": This seminary (...) will take place on the "salle des thèses" of Baulieu, on Tuesday the 8th (from around 9.30pm to $17.00 \mathrm{am}$, with a break to eat around 12.3-14.00 am). It will 
deal with archaeozoology, (...)

Msg. $n^{\circ} 127$ Branch from $n^{\circ} 0124$ 03/04/2003 20:51 GAETAN G. re: SAO...

Hmm... you said there's a seminary on tuesday that begins at 21:30 and that ends at 17:00 ...in the morning(?!?), ...with a break at 12,3 o'clock?!?? Hmm, can I ask you something? ...what are you smoking??? Anyway, that seems interesting and I thank you to remind me that, but I think I'll try to go at 9:30AM, I think it would be more careful!

Msg. $n^{\circ} 128$ Branch from nº 0127 07/04/2003 08:32 MYRIAM M. re: SAO...

sorry for this stupid mistake,but I don't smoke at all!!! it's just a little mistake. It is really at 9.30am...so I'll see you tommorrow!!!

ous observons ici un intéressant exemple de la convergence du linguistique et de l'extra- linguistique (ou encore de la primauté du fond sur la forme). Du point de vue d'un enseignant d'anglais, il s'agit ici manifestement d'une faute d'étourderie. S'il avait à "corriger" cette erreur, l'enseignant attirerait par exemple l'attention de l'étudiante sur l'origine des abréviations am et pm, il sortirait son savoir savant. En revanche, lorsqu'un pair relève l'erreur, il n'en considère que la valeur factuelle, et enfonce le clou ironiquement. Finalement, la correction par les pairs (et par l'ironie) se révèle efficace.

\subsection{Les conversations réflexives}

Dans la publication qui nous sert de guide tout au long de cet exposé, Lamy \& Goodfellow évoquent le concept de conversations réflexives suivies. Il s'agit de conversations dans la mesure où le thème est librement choisi par les étudiants (et non imposé par l'enseignant) ; ces conversations sont réflexives dans la mesure où les interlocuteurs font part de leurs réflexions sur la langue ou l'apprentissage (et - j'ajouterais - sur le dispositif d'apprentissage). Pour Lamy \& Goodfellow, les conversations réflexives sont un lieu idéal d'acquisition de la langue.

\subsubsection{Réemploi de structures}

L'échange suivant est un bel exemple de réemploi spontané de structures, juste après leur présentation au cours d'anglais en présentiel. Le premier message fait allusion au long pseudo- dialogue à propos des Monty Python dont nous avons parlé plus haut (\$ 2.1.3).

Msg. $\mathrm{n}^{\circ} 101$ Branch from $\mathrm{n}^{\circ} 0099$ 21/03/2003 12:39 PHAEDRA B. re: strange disparitions

I thing we should stop as soon as possible (you can just admire this somptuous comparative of equality)to evoque the Monthy Python, otherwise, we should involuntarily bring down attention on them...

Msg. $\mathrm{n}^{\circ} 102$ Branch from $\mathrm{n}^{\circ} 0101$ 24/03/2003 03:05 GAETAN G. re: strange disparitions

And what about the Peoples Front of Judea? You're forgetting them! They have succeeded their mission: the Roman Empire is over now as well as the Popular Front (you can admire my comparative of equality too). But maybe, they're still active in the shade... We have to inquire about this. The sooner we'll know the truth, the faster we could continue our work. (Nice sentence again!) ... 
Tout enseignant de langues a pu constater de tels cas de réemploi de structures, lorsque les apprenants se piquent au jeu, et que leur discours montre qu'ils sont bien conscients qu'il s'agit d'un "jeu"1. Il y a cependant une différence essentielle entre le réemploi de structures dans le cadre des fameux exercices structuraux pratiqués dans les années 1960-1970 - à l'époque de l'audio-oral - et ces réemplois dans le cadre de la conversation réflexive. Dans le cas des exercices structuraux, la mise en situation est pratiquement toujours artificielle, les occurrences de la structure à réemployer sont obligatoires et certaines. Pour suivre la terminologie de van Lier, nous dirions que le discours produit lors de la pratique de tels exercices est non-contingent : "Non-contingent discourse is not anchored within the experiential world (including the here-and-now context) of all participants, nor does it set up expectancies for what is to happen next" (van Lier, 1996 : 183).

En revanche, dans la conversation réflexive, les occurrences des réemplois sont contingentes, toujours au sens de van Lier : "Here it is no longer the case that one person, the teacher, has the agenda, and the students have no option but to follow it. Rather, the agenda is shaped by all participants, and educational reality may be transformed" (op. cit. : 180).

Dans l'échange cité plus haut, nous observons que les apprenants se sont non seulement approprié la structure étudiée en cours, mais qu'ils en revendiquent l'usage libre et non contraint par l'enseignant, à qui ils adressent un message sous la forme d'un clin d'œil. En effet, même si, en surface, les sollicitations de reconnaissance (you can just admire this somptuous comparative of equality, you can admire my comparative of equality too) et la remarque d'autosatisfaction (Nice sentence again!) s'adressent au pair, elles sont en réalité destinées à l'enseignant, dont les étudiants savent bien qu'il lit attentivement tous les messages postés sur ce forum de discussion. Nous ajouterons enfin que de tels réemplois de structure de la langue cible sont un bon exemple de ce que le Cadre européen commun appelle des activités

de nature plus spécifiquement 'pédagogique', [...] fondées sur la nature sociale et interactive 'réelle' et le caractère immédiat de la situation de classe. Les apprenants s'y engagent dans un 'faire-semblant accepté volontairement' pour jouer le jeu de l'utilisation de la langue cible dans des activités centrées sur l'accès au sens (Cadre européen commun, 2001 : 121).

\subsubsection{Réflexion sur le dispositif et ses limites}

41 La conversation qui suit met dans un premier temps en évidence l'influence néfaste de la contrainte que représente l'obligation de participer au forum de discussion. Dans un deuxième temps, toutefois, elle débouche sur un échange fort intéressant sur les vertus du silence.

Msg. $n^{\circ} 142$ Branch from n 0140 09/04/2003 13:29 HELENE M. re: Archaeology can't save the world!

but war are necessary for archeology, the war help the science, in fact, with the napoleonian war we must know a lot of information of egypt, for example (...).

Msg. $\mathrm{n}^{\circ} 144$ Branch from $\mathrm{n}^{\circ} 0142$ 09/04/2003 23:04 JEAN-GUY E. re: Archaeology can't save the world!

On no account we can say that war help for something especially for archaeology. I'm not a pacifist but if war help for something it's for killing more and more people. 
Msg. $n^{\circ} 147$ Branch from n 0142 10/04/2003 08:18 MYRIAM M. re: Archaeology can't save the world!

I refuse to say that war help archaeology! I think war destroys alot of sites, and I don't know how it can help archaeology (...).

Msg. $n^{\circ} 149$ Branch from n 0147 10/04/2003 14:23 HELENE M. re: Archaeology can't save the world!

it was a joke. I just wanted to participate at the forum , but I had nothing of interresting to say. I don't want to create a polemic based on a stupid message! ;-).

Msg. $\mathrm{n}^{\circ} 153$ Branch from nº 0114 11/04/2003 19:26 PHAEDRA B.

Sometimes, when you don't know what to say or if you deliberately know it's not interesting all... you can['t] just keep silent. Silence may sometimes be more eloquent... ;.).

Msg. $n^{\circ} 154$ Branch from nº 0153 12/04/2003 16:24 GAETAN G.

Totally agree :-).

Msg. $n^{\circ} 155$ Branch from $n^{\circ} 0154$ 12/04/2003 16:40 GAETAN G.

I mean, sometimes people write a short message with only a few words, where's the use?? You can search, there isn't any. Even if you read those words several times, trying to find a hidden meaning, you can't cause there are completely useless! On the other hand, some people write long messages with many sentences organized in paragraphs, but without any interesting idea. Here again, where's the use?? I ask you this question cause I have no answer. Maybe, in that last case, that's only because of the pleasure of writing in english. But, is it really a good explanation? I doubt it. That's why I think, if you don't have any interesting things to say, don't open the mouth. And don't put your fingers on the keyboard neither. Because writing for writing (with mistakes, besides), is only a loss of time, for the writer first, but also, for all the students who'll read it till the end. That's all I have to say.

Hélène (Msg. $\mathrm{n}^{\circ}$ 142) fait la proposition que les guerres peuvent en quelque sorte servir la cause de l'archéologie. Cette proposition suscite plusieurs réactions négatives : Msg. $\mathrm{n}^{\circ}$ 144 et Msg. $\mathrm{n}^{\circ} 147$. Il s'ensuit une tentative d'apaisement de la part de l'auteure du message original, assortie d'une explication : Hélène rejette la responsabilité de la situation conflictuelle créée involontairement sur la nécessité de participer au forum même si on n'a rien à dire (Msg. $\left.n^{\circ} 149\right)$. Afin de pallier l'absence d'éléments extralinguistiques de la situation de conversation du dispositif de discussions en ligne, elle ponctue son intervention d'un smiley ;-) équivalant à un clin d'œil.

Phaedra intervient dans la conversation, sur le ton de "la voix de la sagesse" (Msg. $\mathrm{n}^{\circ}$ 153). Argument repris par Gaetan (Msg. $n^{\circ}$ 154) dans un très bref message, développé 15 minutes plus tard... Au passage, il serait intéressant de se demander ce que Gaetan a fait au cours de ces 15 minutes : a-t-il réfléchi à ce qu'il allait écrire ? a-t-il fait un "brouillon" ? Toujours est-il que son message (Msg. $\mathrm{n}^{\circ} 155$ ) est bien tourné et bien envoyé, et qu'il met un terme définitif à la discussion. 


\section{Hyperactifs vs observateurs}

Pour conclure, nous nous intéresserons aux statistiques de consultation et de rédaction des messages sur le forum WebCT au cours de la période considérée (février-mai 2004). Le nombre de sujets ayant participé à l'expérience était de 30 et le nombre total de messages postés a été de 170 environ.

Figure 6 - Nombre de messages lus ( $\min .5 ; \max .173) ; n=30$.

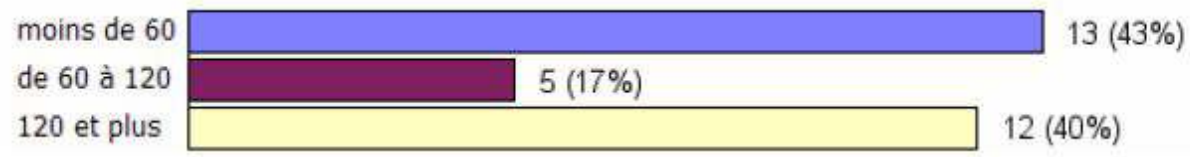

Figure 7 - Nombre de messages originaux postés (min. 0 ; max. 8 ; somme 26$)$; $\mathbf{n}=30$.

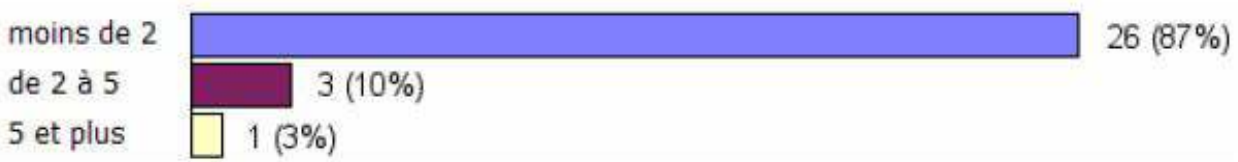

Figure 8 - Nombre de réponses postées $(\min .0 ; \max .30$; somme 104); $\mathrm{n}=30$.

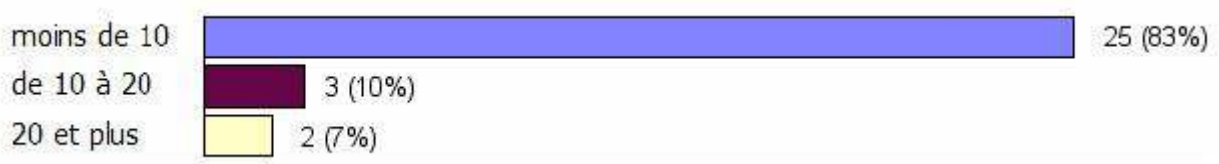

Voici comment interpréter les trois graphiques ci-dessus. Pour chaque question, la population des sujets (étudiants ayant participé à l'expérience) a été subdivisée en 3 classes; pour chaque classe on indique le nombre de sujets qui la compose (et aussi le pourcentage de la population totale). Par exemple sur le graphique de la Figure 6 on peut voir que 13 étudiants (43\%) ont lu moins de 60 messages, 5 (17\%) en ont lu entre 60 et 120, et $12(40 \%)$ en ont lu entre 120 et 173. La comparaison entre les données des trois types d'activité (lecture, envoi de messages originaux et envoi de messages de réponse) appelle les commentaires suivants. On constate une plus grande participation des étudiants à l'activité qui demande le moins de prise de risques, à savoir l'activité de lecture des messages. C'est ainsi que la moitié des étudiants ont lu chacun plus de $50 \%$ des messages postés. En revanche, la répartition de la participation aux deux activités demandant une implication et une prise de risques plus importante suit le classique principe de Pareto ${ }^{2}$. En effet, $79 \%$ des messages originaux ont été postés par $20 \%$ des étudiants et $77 \%$ des messages de réponse ont été postés par $20 \%$ des étudiants, ce qui est assez proche de la proportion $80 / 20$ de Pareto.

Cependant, on aurait tort de penser que seuls les étudiants ouvertement actifs (ceux qui envoient des messages) profitent du dispositif. Les "badauds" (ou lurkers) sont actifs à leur manière, comme le rappelle opportunément ce message qui sera ma conclusion.

Msg. n 166 --- 13/05/2003 15:18 
ELISE B. end of this school year ....retrospective.

So, the end is approaching, how strange it is!so fast! This year we had got a new tool, for expression, exchanges....the webct site. It was very interesting, but when I look, I can see I haven't write a lot...It's a pity isn't it? I have read a lot of message but I'm not particular inspired to write...so, I would thanks people who were more inspired than me, it was very distractiv to read you, and sometimes instructiv too...

Cette étudiante a posté un message original (celui-ci) et deux en réponse à d'autres...

\section{Conclusion}

Ce petit échantillonnage de quelque 180 messages échangés par un groupe d'étudiants sur une période de 4 mois montre que l'outil "Forum de discussion" est un bon facteur de cohésion socio-affective. Ici et là on peut également détecter des traces de conversations réflexives dont on peut supposer qu'elles ont eu un impact positif sur l'acquisition de la langue.

La question reste posée de savoir si - en étant plus présent dans les discussions l'enseignant serait ou non un facteur favorable à cette réflexion sur la langue... Une autre voie qui semble prometteuse pour l'amélioration de la réflexion sur la langue serait l'instauration sur le forum d'un espace spécifique comme les "Travaux pratiques" du dispositif décrit par Lamy \& Goodfellow (2002 : 86). C'est ce que nous avons tenté de faire en 2004 en ouvrant un espace intitulé "Back to English". L'analyse des données recueillies en février-mai 2004 nous permettra de compléter celles qui ont été utilisées pour le présent article et d'améliorer le dispositif du forum de discussion sur WebCT dans les années à venir ${ }^{3}$.

\section{BIBLIOGRAPHIE}

Cadre européen commun de référence pour les langues (2001). Paris : Conseil de l'Europe / Les Éditions Didier. Consulté en mai 2004.

http://culture2.coe.int/portfolio//documents/cadrecommun.pdf

Lamy, Marie-Noëlle, Goodfellow, Robin (1998). "Conversations réflexives dans la classe de langues virtuelle par conférence asynchrone". Apprentissage des Langues et Systèmes d'Information et de Communication, (Alsic), vol. 1, n 2. pp. 81-101. Consulté en mai 2004. http://archiveedutice.ccsd.cnrs.fr/docs/00/00/16/90/HTML/index.html

Rézeau, Joseph (2001). Médiatisation et médiation pédagogique dans un environnement multimédia : Le cas de l'apprentissage de l'anglais en Histoire de l'art à l'université. Thèse pour le Doctorat, université Victor Segalen-Bordeaux 2, France. Consulté en mai 2004. http://archive-edutice.ccsd.cnrs.fr/ edutice-00000222 (theseRezeau.pdf).

van Lier, Leo (1996). Interaction in the Language Curriculum - Awareness, Autonomy \& Authenticity. London: Longman. 


\section{Sites}

Wikipedia (nd). Encyclopédie en ligne. Consulté en octobre 2004. http://en.wikipedia.org/wiki/ Pareto_principle

\section{NOTES}

1. Nous analysons des exemples de ce type de réemplois " en situation " dans notre thèse (Rézeau, 2001 : pp. 544- 545).

2. Principe de Pareto ou principe du $80 / 20$ selon lequel $80 \%$ des résultats proviennent de $20 \%$ des activités réalisées. (cf. site Wikipedia).

3. Au moment où nous écrivons ces lignes, nous apprenons que notre université a décidé d'abandonner la plate- forme WebCT à la rentrée 2005, en raison de son coût jugé prohibitif. WebCT sera remplacé par une plate-forme alternative, probablement Moodle, qui dispose également d'un bon outil de forum de discussion.

\section{RÉSUMÉS}

Au moment où nombre d'institutions de l'enseignement supérieur en France mettent en place des cours en ligne, on peut s'interroger sur la pertinence des nouveaux outils d'apprentissage dits collaboratifs tels que les forums de discussion et sur leur apport spécifique par rapport au cours en présentiel. Dans le cadre de l'apprentissage d'une langue étrangère par des étudiants spécialistes d'autres disciplines, on peut espérer que l'outil "forum de discussion" offrira aux apprenants la possibilité non seulement d'être exposés à des stimuli en langue cible mais surtout de produire eux-mêmes de la langue. Après avoir présenté le cadre plus général dans lequel se situe l'expérience menée avec nos étudiants d'archéologie à l'université de Rennes 2, nous suivons la typologie proposée par Lamy \& Goodfellow (1998) pour chercher à savoir si le dispositif que nous avons mis en place suscite des dialogues réflexifs, considérés par ces auteurs comme le type d'échanges le plus favorable à l'acquisition de la langue étrangère.

As more and more French universities set up online courses, the relevance of so-called collaborative learning tools such as asynchronous electronic conferencing and their specific contribution compared to face-to-face teaching can be questioned. In an English for Specific Purposes (ESP) situation, it is expected that an online discussion forum will offer students the opportunity not only to be submitted to target language stimuli but also to produce language. This article first describes the general framework of the experiment led with our students of the English for Archaeology Course at the university of Rennes 2. We then adopt the typology suggested by Lamy \& Goodfellow (1998) in order to find out whether the Discussion Forum we have set up is likely to give rise to the "reflective conversation" type of exchanges deemed by these authors to be the most favourable to language acquisition. 
INDEX

Mots-clés : apprentissage à distance, forum de discussion électronique, stratégies d'apprentissage, interaction

Keywords : distance learning, interaction, strategies, asynchronous conferencing, forum, discussion group

\section{AUTEUR}

\section{JOSEPH RÉZEAU}

Joseph Rézeau est maître de conférences en anglais de spécialité à l'université Rennes 2. Il a soutenu en 2001 une thèse intitulée : "Médiatisation et médiation pédagogique dans un environnement multimédia". Il s'intéresse principalement à la conception de dispositifs d'apprentissage multimédias et interactifs, ainsi qu'à l'analyse de la trace du parcours des apprenants utilisateurs de tels dispositifs.

Département d'anglais, Université Rennes 2, F-35043 RENNES CEDEX joseph.rezeau@uhb.fr http://rezeau.org 